\title{
Effect of vitamin K2 on the development of stress-induced osteopenia in a growing senescence-accelerated mouse prone 6 strain
}

\author{
HIRONOBU KATSUYAMA $^{1}$, SHIGEKO FUSHIMI ${ }^{1,2}$, KUNIKAZU YAMANE $^{1}$, YOKO WATANABE $^{3}$, \\ KOICHIRO SHIMOYA ${ }^{4}$, TOSHIKO OKUYAMA ${ }^{5}$, MIDORI KATSUYAMA ${ }^{6}$, \\ KIYOFUMI SAIJOH $^{6}$ and MASAFUMI TOMITA ${ }^{5}$ \\ ${ }^{1}$ Department of Public Health, Kawasaki Medical School, Kurashiki, Okayama 701-0192; \\ ${ }^{2}$ Department of Oral Pathology and Medicine, Okayama University School of Medicine, \\ Dentistry and Pharmaceutical Sciences, Okayama 700-8525; Departments of ${ }^{3}$ Natural Sciences, \\ ${ }^{4}$ Obstetrics and Gynecology and ${ }^{5}$ Medical Toxicology, Kawasaki Medical School, Kurashiki, Okayama 701-0192; \\ ${ }^{6}$ Department of Hygiene, Kanazawa University School of Medicine, Kanazawa, Ishikawa 920-8640, Japan
}

Received April 8, 2014; Accepted June 1, 2015

DOI: $10.3892 / \mathrm{etm} .2015 .2621$

\begin{abstract}
Vitamin K2 (VK2) has been used as a therapeutic agent for osteoporosis, since it has been suggested to be able to reduce the frequency of fractures by improving bone quality; however, bone turnover is strictly regulated by various cytokines and hormones. In the present study, the effect of menaquinone-4 (MK-4) on bone turnover was investigated using the senescence-accelerated mouse prone 6 (SAMP6) strain. Since water-immersion restraint stress (WRS) causes a significant decrease in bone mineral density (BMD), WRS was used as the bone resorption model in the SAMP6 strain. Six-week-old SAMP6 male mice were divided into the following three groups: Control, WRS and WRS + MK-4. WRS was performed for $6 \mathrm{~h}$ per day, 5 times a week, for 4 weeks. Following WRS, MK-4 (30 mg/kg) was injected subcutaneously 3 times a week for 4 weeks. No growth retardation was observed in the WRS groups as compared with the control group. In the WRS groups, the BMD was significantly lower than that in the control group. The levels
\end{abstract}

Correspondence to: Professor Hironobu Katsuyama, Department of Public Health, Kawasaki Medical School, 577 Matsushima, Kurashiki, Okayama, 701-0192, Japan

E-mail:katsu@med.kawasaki-m.ac.jp

Abbreviations: MK-4, menaquinone-4; BMD, bone mineral density; SAMP6, senescence-accelerated mouse prone 6; VK2, vitamin K2; WRS, water-immersion restraint stress; ALP, alkaline phosphatase; OCN, osteocalcin; TRACP, tartrate-resistant acid phosphatase; CTX, C-terminal telopeptides of type I collagen; Oc, osteoclast; $\mathrm{Ob}$, osteoblast

Key words: senescence-accelerated mouse prone 6, water-immersion restraint stress, vitamin $\mathrm{K} 2$, bone histomorphometry, bone resorption, bone formation of bone formation and resorption markers were increased in the WRS groups, indicating that WRS reduced the BMD by promoting high bone turnover. A bone histomorphometrical examination showed that the trabecular $(\mathrm{Tb})$ bone mass in the secondary spongiosa at the distal femur was significantly reduced in the WRS mice, and this reduction was abrogated by MK-4 treatment. Specifically, the Tb bone reduction was caused by the activation of osteoclasts (Ocs), and Oc activity was suppressed by MK-4. The number of osteoblasts and the mineral apposition rate were significantly increased in the WRS and WRS + MK-4 mice, suggesting that WRS triggered a significantly higher mineral apposition rate. These results indicate that MK-4 can induce recovery from the bone mineral loss caused by WRS treatment. Further studies are required to clarify the association between bone quality and MK-4.

\section{Introduction}

Supplementation of menaquinone (MK)-7, a subtype of vitamin K2 (VK2), has been found to significantly improve the age-related decline in bone health and the bone mineral density (BMD) at the lumber spine and femoral neck (1). Among the VK2 molecules, MK-4 (VK2 with 4 isoprene units) is known to contribute to high $\gamma$-carboxylation activity and exhibit the most potent biological activity of the VK2s (2). Previous meta-analyses have demonstrated the anti-fracture effect of VK2 in patients with a high risk of fracture $(3,4)$. In a clinical study, VK2 administration was not shown to result in an increase in BMD, but the frequency of bone fracture was found to be significantly reduced (1). Ovariectomized rats show significant bone loss, which VK2 prevents by inhibiting bone resorption and osteoclast (Oc) formation, as determined by bone histomorphometry (5). VK2 has been shown to exhibit the novel function of binding to the pregnane $\mathrm{X}$ receptor (PXR), and PXR-dependent biological functions have been demonstrated in bone (6); however the biological effect of VK2 remains controversial, 
since $\mathrm{Fu}$ et al (7) reported that $\mathrm{VK} 2$ failed to prevent bone loss in ovariectomized rats.

Water-immersion restraint stress (WRS), which involves both psychological and physical stress, is widely used to induce reproducible stress ulcers in experimental animals (8-12). WRS consists of immobilization and cold stress, in which the exposure temperature is usually $24^{\circ} \mathrm{C}$. Rats subjected to $6 \mathrm{~h}$ of WRS exhibit disruption of the non-enzymatic antioxidant defense systems in the brain and marked increases in the serum concentrations of adrenocorticotropic hormone and corticosterone (13); however, the effect of stress on bone mass and bone turnover is still debated.

Senescence-accelerated mouse (SAM) strains are developed by means of repeated inbreeding, using shortness of lifespan and aging speed as indices (14). All SAM prone (SAMP) strains are short-lived and exhibit accelerated senescence. SAMP strains are categorized into 11 groups according to the senile diseases that they develop naturally, such as senile amyloidosis (SAMP1, 2, 7 and 11), temporomandibular disorders (SAMP3), senile osteoporosis (SAMP6), learning and memory impairments (SAMP8 and 10) and cataract (SAMP9) (15-17). The SAMP6 strain presents decreasing BMD from the age of 16 weeks, and has a lifespan of $\sim 32$ weeks (18). In order to assess the effects of various drugs affecting BMD, additional approaches to reduce BMD must be taken in the SAMP6 strain.

In the present study, the effect of WRS on BMD was estimated and the histomorphometrical phenotype of bone resorption and formation in the proximal femur metaphysis of SAMP6-strain mice was characterized. Furthermore, the effect of MK-4 on WRS-induced bone loss was investigated using bone histomorphometry, bone densitometry and biomarkers of bone turnover.

\section{Materials and methods}

Animal experiments. Five-week-old SAMP6 males were purchased from Japan SLC, Inc. (Hamamatsu, Japan). The animals were given a normal rodent chow and tap water and were acclimated to the conditions for 1 week. The mice were then divided into the following three groups ( $n=6$ per group): Control, WRS and WRS + MK-4. WRS was performed for $6 \mathrm{~h}$ per day, 5 times a week, for 4 weeks. Following WRS, MK-4 (30 mg/kg; Eisai Co., Ltd., Tokyo, Japan), a VK2 molecule, was injected subcutaneously 3 times/week, while the control and WRS mice were injected with saline solution as a vehicle. The WRS method has been previously described in detail (19). Briefly, the mice were restrained in a punctured $50-\mathrm{ml}$ conical centrifuge tube and immersed vertically to the level of the xiphoid process in a $24 \pm 1^{\circ} \mathrm{C}$ water bath. To assess bone formation, the mice were double-labeled with subcutaneous injections of $20 \mathrm{mg} / \mathrm{kg}$ tetracycline hydrochloride and $10 \mathrm{mg} / \mathrm{kg}$ calcein (Sigma-Aldrich Corp., St. Louis, MO, USA), 5 and 2 days before sacrifice, respectively. The body weight of each animal was measured every other day until the last day of MK-4 administration. Mice were placed in metabolic cages and urine samples were collected $16 \mathrm{~h}$ after the final administration. Urine samples were stored at $-80^{\circ} \mathrm{C}$ until analysis. One day after the final MK-4 administration, the mice were anesthetized using isoflurane, and blood samples were collected from the abdominal aorta, the mice died due to excessive loss of blood. The blood samples were centrifuged at 3,200 x g for $10 \mathrm{~min}$ at room temperature to separate the serum, which was then stored at $-80^{\circ} \mathrm{C}$. Following the collection of the blood samples, the bilateral femurs were removed, cleaned from soft tissues and fixed in $70 \%$ ethanol. The experimental protocol was approved by the Animal Research Committee of the Kawasaki Medical School (Kurashiki, Japan; 11-031).

Trabecular ( $T b$ ) BMD. The fixed right femoral bones were analyzed using an X-ray Computed Tomography system for small experimental animals (LaTheta LCT-200; Aloka Co., Ltd, Osaka, Japan). Each bone was placed horizontally inside a tube and scanned using a $96-\mu \mathrm{m}$ voxel. The scan line was adjusted using the scout view. The Tb BMD in the secondary spongiosa was measured from the growth plate to $3 \mathrm{~mm}$ proximal at the femoral distal end. The data were quantified using the automated image analysis software supplied with the device (LCT-200F1, v.3.22; Aloka Co., Ltd.).

Analysis of bone turnover biochemical markers. Urinary $\mathrm{Ca}^{2+}$ concentration and serum alkaline phosphatase (ALP) activity were determined using an autoanalyzer (Hitachi 7180; Hitachi, Ltd., Tokyo, Japan). The total serum protein concentration was measured using a refractometer (Atago Co., Ltd., Tokyo, Japan). Serum levels of Gla-osteocalcin (OCN) and tartrate-resistant acid phosphatase (TRACP) 5 b were determined using a mouse Gla-OCN Competitive Enzyme Immunoassay (EIA) kit (Takara, Inc., Kyoto, Japan) and a mouse TRACP assay kit (Immunodiagnostic Systems, Inc., Fountain Hills, AZ, USA), respectively, according to the manufacturers' instructions. The concentration of C-terminal telopeptides of type I collagen (CTX) in the urine was determined using a commercial EIA kit (Immunodiagnostic Systems, Inc.), according to the manufacturer's instructions, and the levels were corrected against the creatinine $(\mathrm{Cr})$ concentration. The $\mathrm{Cr}$ level in the collected samples was measured by SRL Inc. (Tokyo, Japan).

Bone histomorphometry. Bone histomorphometry was performed on the secondary spongiosa of the left femoral distal end of samples at the Ito Bone Histomorphometry Institute (Niigata, Japan). The coronal view of the femoral distal end was observed using Villanueva Bone Staining. Bone histomorphometrical examination was used to evaluate the tissue volume (TV, $\left.\mu \mathrm{m}^{2}\right)$, bone volume $\left(\mathrm{BV}, \mu \mathrm{m}^{2}\right)$, bone surface (BS, $\mu \mathrm{m})$, single-labeled surface, double-labeled surface (dLS, mm), $\mathrm{Tb}$ thickness (Tb.Th, $\mu \mathrm{m}$ ) and the number of osteoclasts and osteoblasts (N.Ocs and N.Obs, respectively). Obs were further classified into type II-IV according to morphological classification criteria (20): Type II, classical cuboidal or columnar with adjacent nuclear clear zone, type III, intermediate without adjacent nuclear clear zone and type IV, cytoplasm-extremely thin (most mature population) (21). Type I Obs cannot be detected by microscopy. The following parameters were then estimated from the primary parameters: BV/TV (\%), osteoid surface (OS)/BS (\%), eroded surface (ES)/BS (\%), N.Ocs/BS $(\mathrm{N} / \mathrm{mm})$, surface area of Ocs (Ocs.S)/BS (\%) mineral apposition rate (MAR, $\mu \mathrm{m} /$ day), Obs.S/BS (\%) and N.Obs/BS (N/mm). Standard bone histomorphometrical nomenclature, symbols and units were used as described in the report by the American 
A
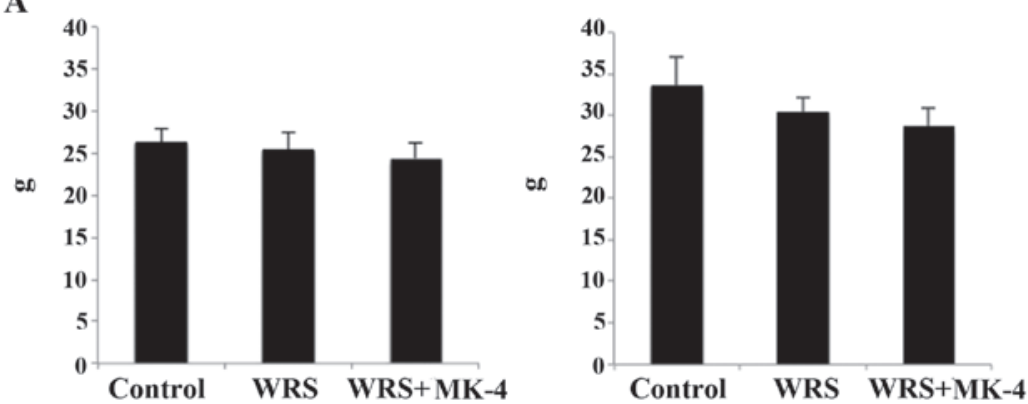

B

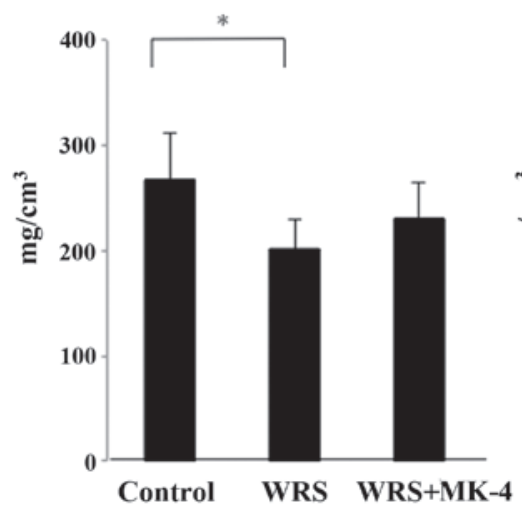

C

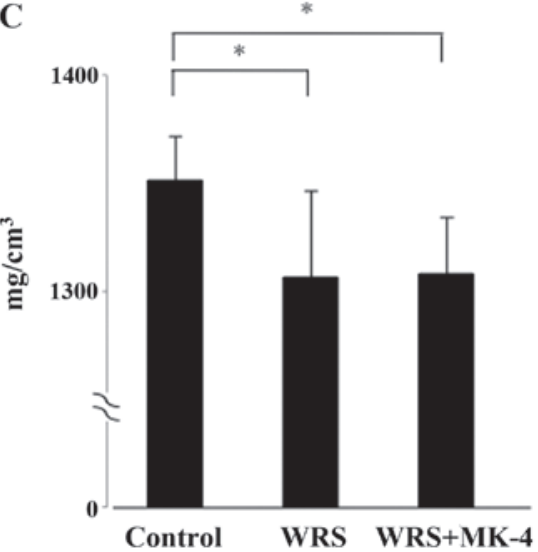

Figure 1. Growth of SAMP6 mice and effects of WRS on femoral BMD. (A) The initial body weight of 6-week-old (left) and 10-week-old (right) SAMP6 mice was measured. No growth retardation was observed. (B and C) Effects of WRS on (B) trabecular and (C) cortical BMD. Data are expressed as the mean \pm standard deviation of 6 animals. Analysis of variance followed by the Tukey-Kramer post hoc test was performed to compare data among the three groups. $\mathrm{P}<0.05$. SAMP6, senescence-accelerated mouse prone 6; WRS, water-immersion restraint stress; MK-4, menaquinone-4; BMD, bone mineral density.

Society for Bone and Mineral Research Histomorphometry Nomenclature Committee (22).

Statistical analysis. Data are expressed as the mean \pm standard deviation. In order to compare the differences between the control, WRS and WRS + MK-4 groups, a one-way analysis of variance (ANOVA), followed by the Tukey-Kramer post hoc test, was employed using JMP 10 software (SAS Institute Inc., Cary, NC, USA). $\mathrm{P}<0.05$ was considered to indicate a statistically significant difference.

\section{Results}

Growth of SAMP6 mice and effect of WRS on BMD. No significant differences were observed in either the initial or final body weights among the groups, despite the fact that the final body weights of the WRS groups had a tendency to be lower than those of the control group (Fig. 1A). In addition, the femoral lengths were $15 \mathrm{~mm}$ in all groups, and no statistically significant differences were observed. Both the $\mathrm{Tb}$ and the cortical BMDs were significantly decreased in the WRS mice compared with those in the control mice (Fig. 1B and C).

Effect of MK-4 on biochemical markers of bone turnover following WRS. In the WRS group, levels of serum ALP, a bone formation marker, were significantly higher than those in the control; however, MK-4 had no effect on serum ALP in the WRS mice (Fig. 2A). No significant differences were identified in the Gla-OCN levels among the groups (Fig. 2B); however, $\mathrm{Ca}^{2+}$ excretion in the urine was significantly higher in the WRS group than that in the control group, and this excretion was suppressed following the administration of MK-4 (Fig. 2C). The levels of urinary CTX, a degradation product of collagen, were significantly higher in the WRS and WRS + MK-4 groups than those in the control (Fig. 2D); however, no significant differences were identified in TRACP $5 b$, a bone resorption marker (Fig. 2E).

Microscopic observation of the distal femurs of SAMP6 mice. The sagittal view of the left distal femurs was observed using Villanueva Bone Staining (Fig. 3A). Microscopic observation showed that the growth plate and primary spongiosa were thin in all groups. These findings suggested that SAMP6 mice were senescent at 10 weeks of age. When the SAMP6 mice were exposed to WRS, the quantity of $\mathrm{Tb}$ bone in the secondary spongiosa of the distal femur decreased. The $\mathrm{Tb}$ bone mass was recovered when MK-4 was administered. The Tb.Th was significantly lower in the WRS group than that in the control group, and this reduction was attenuated in the WRS + MK-4 group (Fig. 3B).

Bone resorption in the $\mathrm{Tb}$ bone of SAMP6 mice. The bone resorption area was first evaluated using bone histomorphometry (Fig. 4A). The ES/BS percent age was significantly higher in the WRS group than that in the control group (Fig. 4A and B) and was effectively reduced by MK-4. 
A

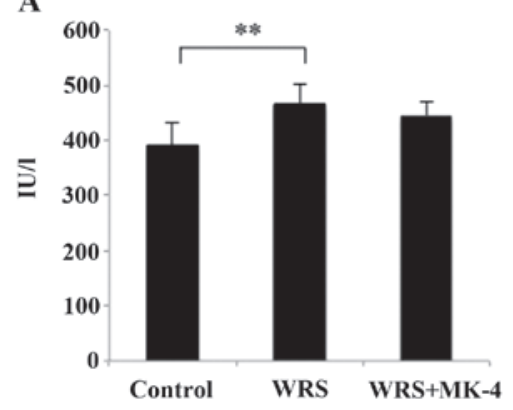

B

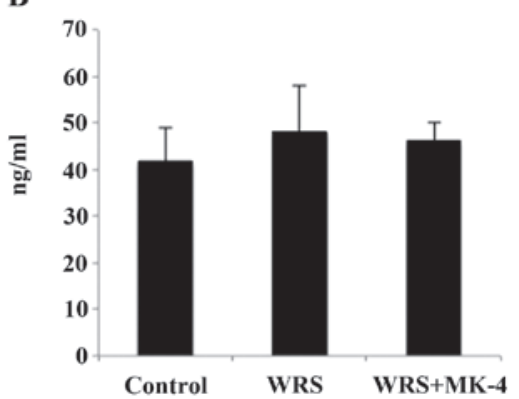

C

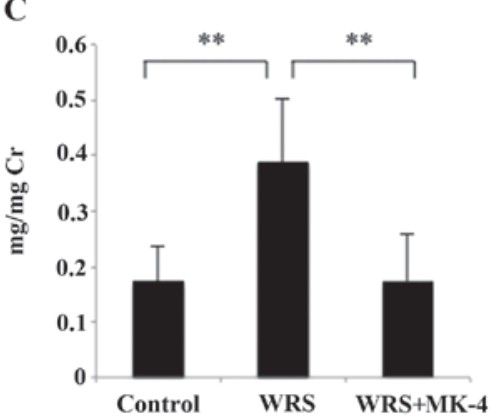

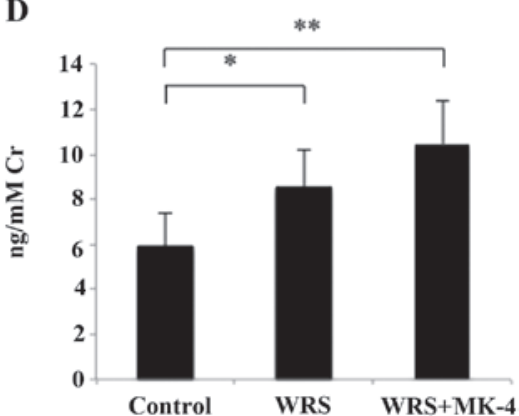

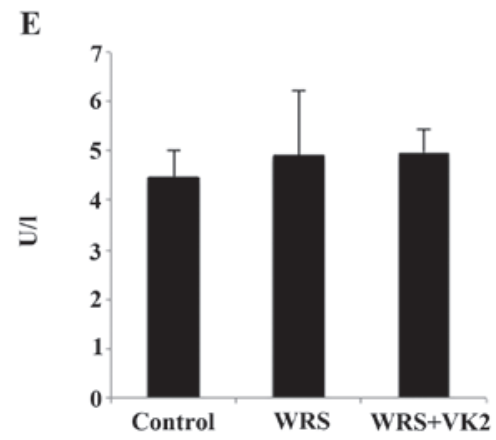

Figure 2. Effects of WRS and MK-4 on bone turnover markers. The bone formation markers (A) alkaline phosphatase and (B) Gla-osteocalcin and bone resorption markers (C) urinary $\mathrm{Ca}^{2+}$, (D) C-terminal telopeptide of type I collagen and (E) tartrate-resistant acid phosphatase 5b were measured. Data are presented as the mean \pm standard deviation. ${ }^{*} \mathrm{P}<0.05$ and ${ }^{* *} \mathrm{P}<0.01$. WRS, water-immersion restraint stress; MK-4, menaquinone-4; Cr, creatinine.

A

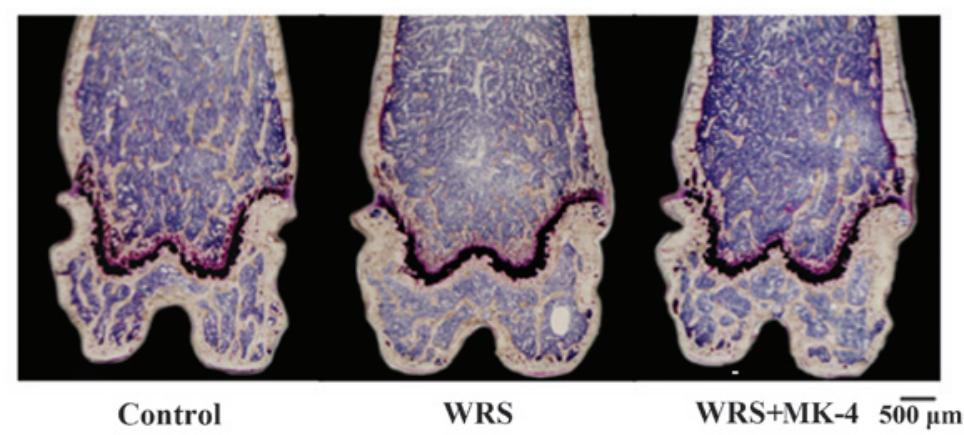

B

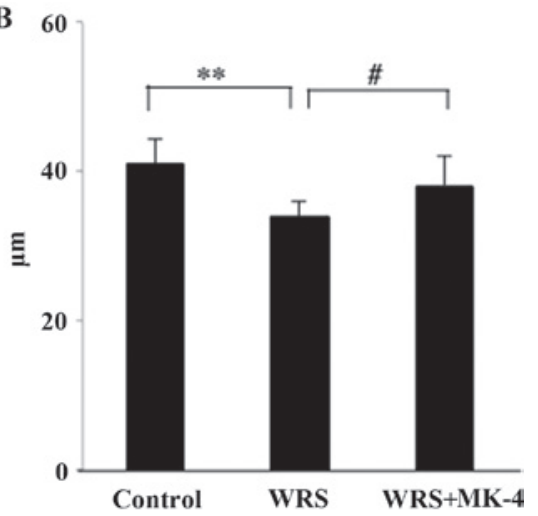

Figure 3. Comparisons of trabeculae among control, WRS and WRS + MK-4 groups. (A) Trabeculae in the secondary spongiosa of distal femurs were observed using Villanueva Bone Staining. (B) Trabecular thickness was compared among the three groups. Data are expressed as the mean \pm standard deviation.** $\mathrm{P}<0.01$ and ${ }^{\#} \mathrm{P}<0.10$. WRS, water-immersion restraint stress; MK-4, menaquinone-4.

The Ocs.S/BS percentage was significantly higher in the WRS groups than that in the control group (Fig. 4C). Furthermore, the number of multinucleated Ocs was significantly higher in the WRS group than that in the control group (Fig. 4D), an increase that was not observed in the WRS + MK-4 group. No significant differences were observed in the number of mononucleated Ocs among these groups. 
A

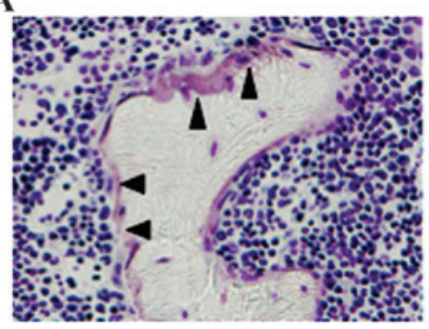

Control

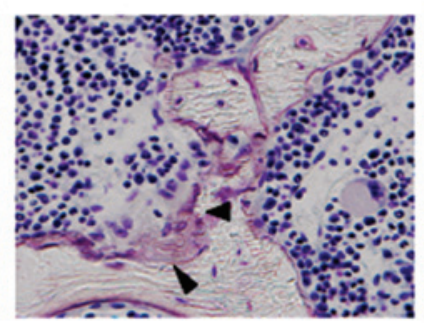

WRS

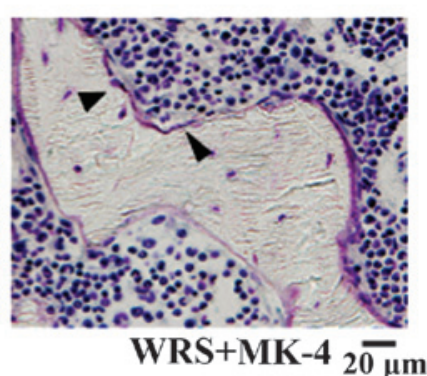

D
B

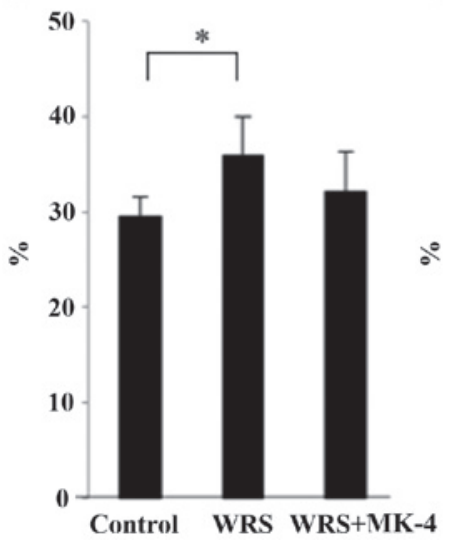

C

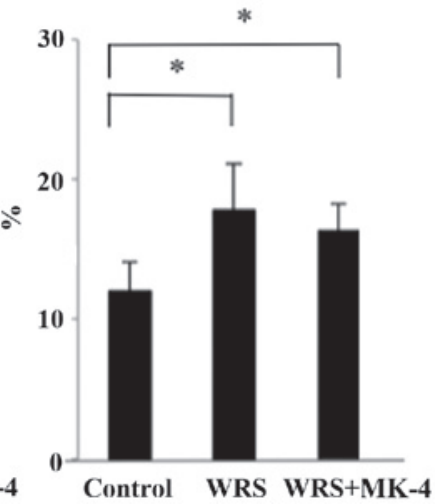

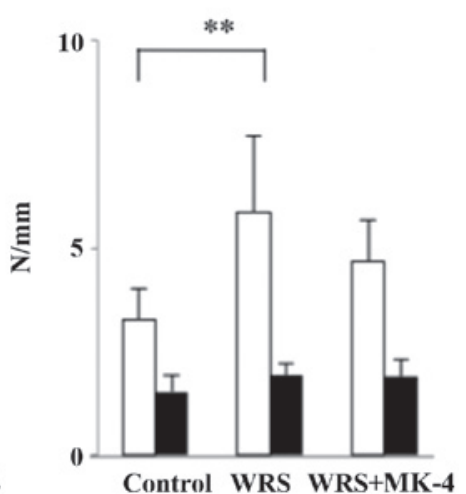

Figure 4. Comparisons of bone resorptive parameters among the control, WRS and WRS + MK-4 groups. (A) Rough surface shows the resorption area, indicated by arrows. (B) Eroded surface/bone surface percentages, (C) Oc surface/bone surface percentages and (D) numbers of multinucleated Ocs (white bar) and mononucleated Ocs (black bar) were compared among the three groups. Data are expressed as the mean \pm standard deviation. $\mathrm{P}<0.05$ and ${ }^{* *} \mathrm{P}<0.01$. WRS, water-immersion restraint stress; MK-4, menaquinone-4; Oc, osteoclast.

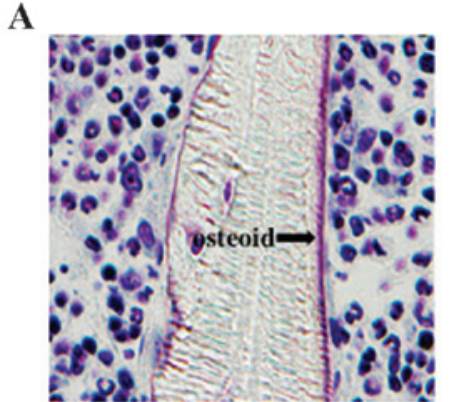

Control

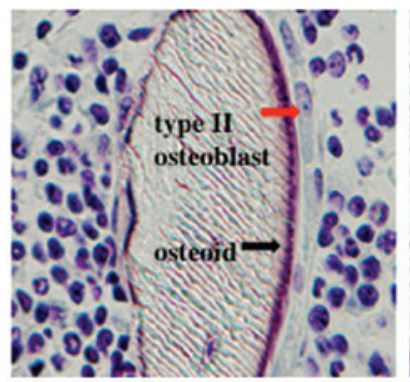

WRS

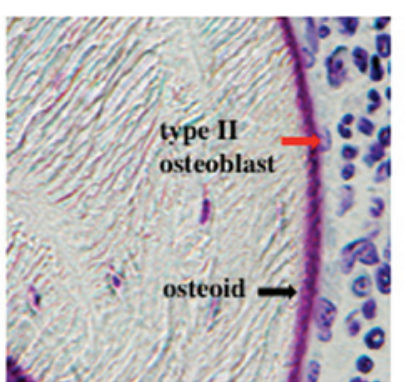

WRS+MK-4 $10 \bar{\mu} \mathrm{m}$
B

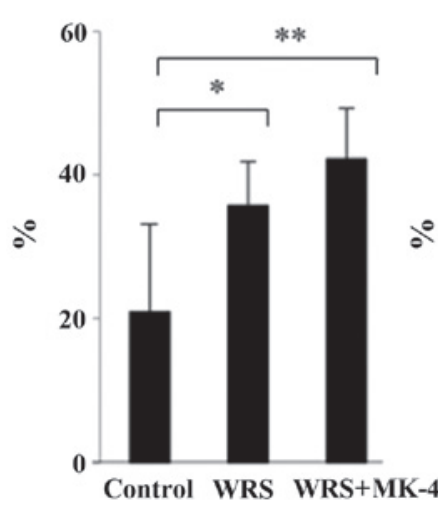

C

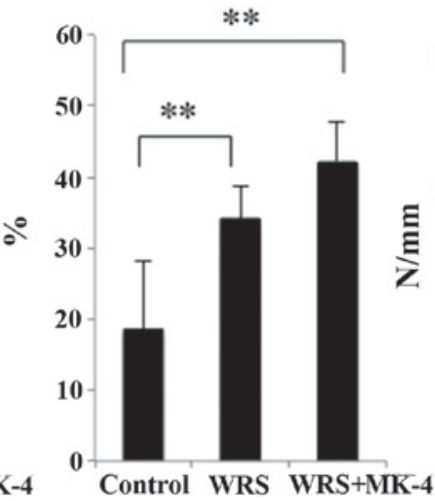

D

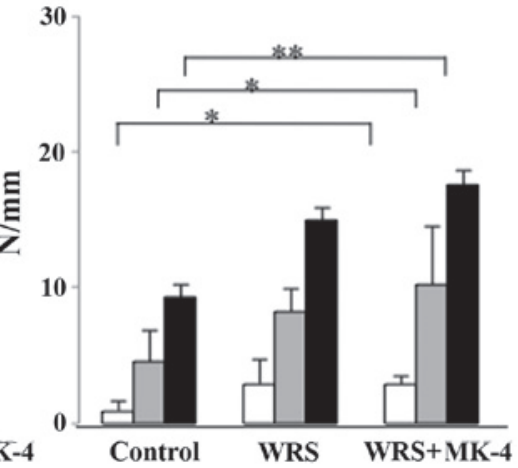

Figure 5. Measurements of osteoid width and Ob formation. (A) Microscopic observation of osteoid width and types of Ob. A red arrow indicates the type II cuboidal Ob. (B) Osteoid surface/trabecular bone surface percentages, (C) Ob surface/bone surface percentages and (D) numbers of type II (white bar), type III (grey bar) and type IV (black bar) Obs were compared among the three groups. Data are expressed as the mean \pm standard deviation. $\mathrm{P}<0.05$ and ${ }^{* *} \mathrm{P}<0.01$. WRS, water-immersion restraint stress; MK-4, menaquinone-4; Ob, osteoblast. 


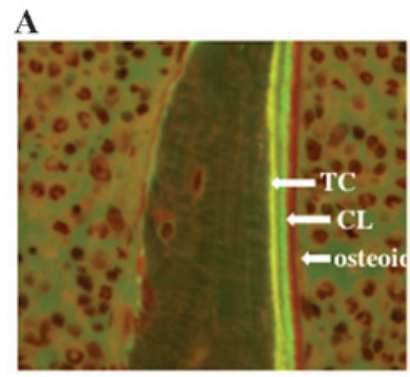

Control

B

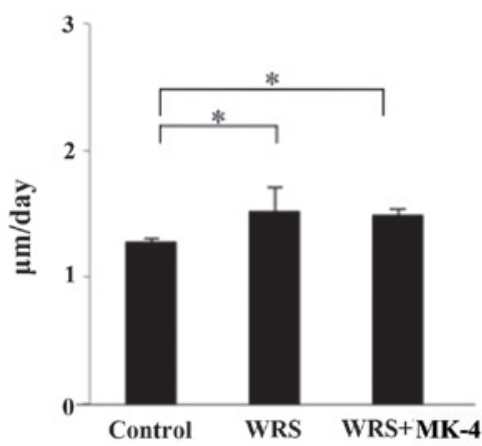

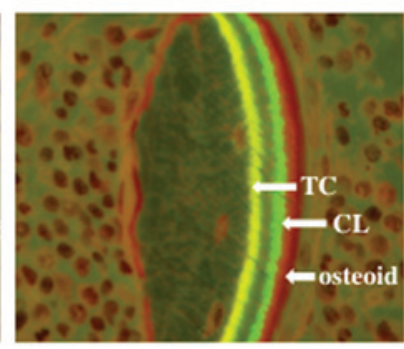

WRS

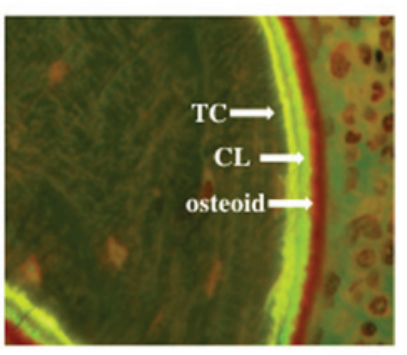

WRS+MK-4 $10 \overline{\mu m}$

C

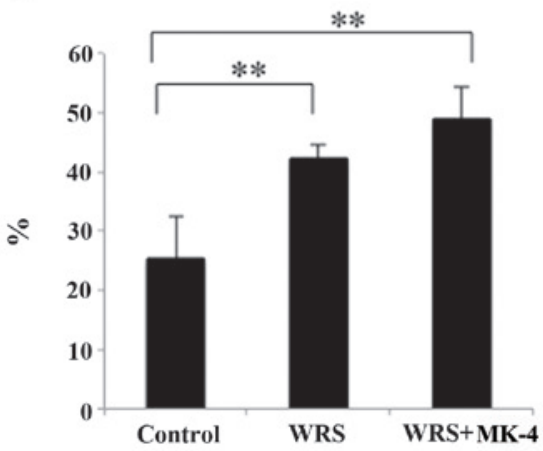

D

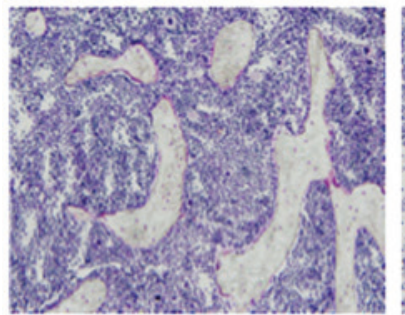

Control

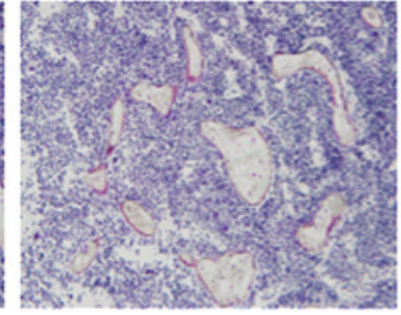

WRS

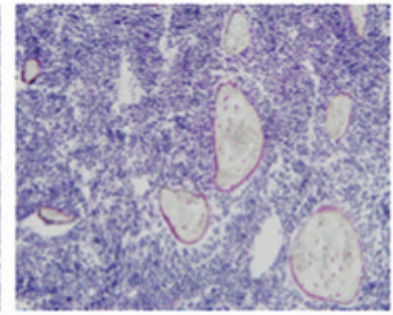

WRS+MK-4

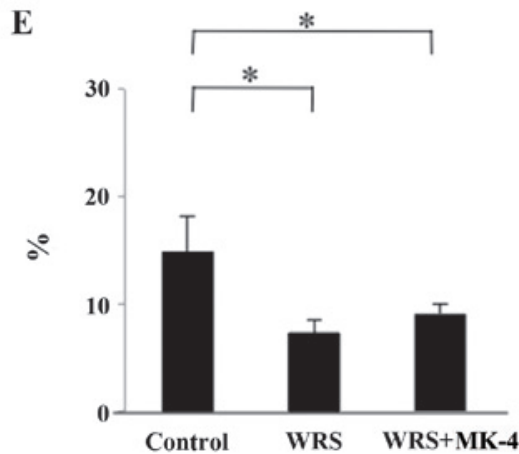

Figure 6. Mineralization parameters induced by WRS with/without MK-4. (A) Mineral apposition of trabecular bone was measured by double staining with TC (yellow) and CL (green). The interval between the TC and CL injections was 2 days, and the distances between CL and TC layers were used to estimate the MAR. (B) MAR, (C) double-labeled surface/bone surface percentages and (D and E) bone volume/tissue volume were compared among the three groups. Data are expressed as the mean \pm standard deviation. ${ }^{*} \mathrm{P}<0.05$ and ${ }^{* *} \mathrm{P}<0.01$. TC, tetracycline; CL, calcein; MK-4, menaquinone-4; WRS, water-immersion restraint stress; MAR, mineral apposition rate.

Bone formation in the Tb bone of SAMP6 mice. Microscopic observation showed the osteoid width to be thicker in the WRS + MK-4 group than that in the control group (Fig. 5A). The $\mathrm{OS} / \mathrm{BS}$ percentages in the WRS groups were significantly higher than those in the control group (Fig. 5B), indicating that WRS induced bone formation. Furthermore, the Obs.S/BS percentages in the WRS groups were significantly higher than those in the control group (Fig. 5C). Although no difference was observed between the WRS and WRS + MK-4 groups, all the numbers of all Ob types were significantly increased in the WRS + MK-4 group compared with the control group (Fig. 5D). These results indicated that MK-4 increased the N.Obs. In combination with its effect on Ocs, WRS may cause both bone resorption and formation, resulting in increased bone turnover. In addition, MK-4 may stimulate recovery from WRS-induced bone loss.

$M A R$ and $B V$. Since tetracycline and calcein were used for double staining, the time interval between the injections of 
the two labels enabled the determination of the MAR, with a wide physical distance between the labeled layers indicating rapid mineralization. The WRS and WRS + MK-4 groups exhibited a significantly higher MAR than the control group (Fig. 6A and B), and the dLS/BS was significantly higher in the WRS and WRS + MK-4 groups compared with that in the control group (Fig. 6C). These results indicated that WRS promoted bone formation and MK-4 also appeared to promote bone mineralization.

Microscopic observation of the bones showed that the $\mathrm{Tb}$ bones of all groups were small, rounded and isolated (Fig. 6D). This phenomenon was considered characteristic of the SAMP6 strain. In addition, the Tb bone shape in the WRS group was small and scattered compared with the control and WRS + MK-4 groups, and the BV/TV percentage was significantly lower in the WRS and WRS + MK-4 groups than that in the control group (Fig. 6E).

\section{Discussion}

The SAMP6 strain shows the characteristic Tb bone shape in bone histomorphometry, and WRS successfully reduces BV/TV. The SAMP6 strain starts losing bone at 16 weeks and exhibits BV and BMD loss at 28 weeks (18); however, ovariectomy of female SAMP6 mice does not lead to an increase in osteoclastic activity or a further decrease in bone mass, since the SAMP6 strain is a hormone-independent model of osteoporosis (23). Despite attempts in the present study to induce bone loss by ovariectomy of female SAMP6 mice, the females showed no bone loss at 12 weeks (data not shown). Thus, SAMP6 males were used, since they are hormonally stable in adolescence compared with SAMP6 females.

WRS is often used as a stress treatment to induce stress response syndromes in animals $(8-13,24)$. In the present study, WRS successfully reduced the BMD via a high bone turnover rate; WRS induced an increase in bone resorption and formation. There are several mouse models for the reduction of BMD, such as ovariectomy of female mice (25), administration of soluble receptor activator of nuclear factor $\kappa \mathrm{B}$ ligand (RANKL) (26) and tail suspension (27). Ovariectomy leads to BMD loss for 8 weeks, but requires specific surgical skills, while the intraperitoneal injection of soluble RANKL reduces BMD for only $50 \mathrm{~h}$; however, soluble RANKL is expensive and difficult to obtain. Tail suspension leads to a reduction of BMD for 14 days, caused by immobilization. The type of bone loss caused by WRS is different from immobilization-induced bone loss, since immobilization leads to incremental bone resorption but to a decrease in bone formation (27). In the present study, WRS did not trigger growth retardation, and bone reduction was observed after a 4 -week treatment. Thus, WRS could be a model of stress response and osteopenia in the SAMP6 strain.

Since VK2 consists of 14 isoprene units and MK-4 is the most potent form of $\mathrm{VK} 2, \mathrm{MK}-4$ was administered subcutaneously to SAMP6 mice as VK2 treatment. The biological function of VK2 is associated with anticoagulation and $\gamma$-carboxylation in various proteins (28). Most VK-dependent enzymes are involved in the hemostatic process and are associated with bone metabolism. OCN and matrix Gla protein are two major Gla proteins in the bone (29), but their benefi- cial effects on the bone remain unclear. Studies have shown that VK2 attenuates Tb bone loss in glucocorticoid-treated rats (30) and prevents bone loss by inhibiting bone resorption in ovariectomized rats (5). In vitro studies revealed that VK2 influences Ob differentiation (31), binds to the steroid xenobiotic receptor (6) and accelerates bone mineralization (32). In addition, a clinical study demonstrated that VK2 administration effectively reduced the osteoporotic fracture incidence (1). Furthermore, VK2 induces Oc apoptosis and reduces the bone resorption area (33). Based on these reports, VK2 may maintain bone strength through the activation of osteoblastic function and the suppression of osteoclastic function. The bone histomorphometrical examination in the present study showed that MK-4 attenuated WRS-induced Tb bone loss by inhibiting Oc activity and increasing Ob activity. Furthermore, the $\mathrm{Tb}$ bone shape in the WRS mice was quite different from that in the WRS + MK-4 mice. Despite the fact that bone strength was not measured, the aforementioned results indicate that MK-4 may improve BMD.

The present study had several limitations. First, although MK-4 was administered subcutaneously to mice, the absorption of MK-4 was not considered and the serum concentration of MK-4 was not measured. Secondly, the SAMP6 strain was used for all experiments; the results obtained in this study could therefore be strain-specific phenomena.

In conclusion, WRS effectively reduced BMD in a short period of time in the SAMP6 mice. Furthermore, MK-4 treatment was efficient in recovering the WRS-induced bone mineral loss, indicating its effect on the suppression of Oc function and the increase in $\mathrm{Ob}$ function. Further studies are required in order to clarify the association between MK-4 administration and bone quality using a different mouse strain.

\section{Acknowledgements}

This study was supported by Grants-in-Aid for Scientific Research (KAKENHI; grant no. 22500684). The authors would like to acknowledge Editage for their English editing service.

\section{References}

1. Knapen MH, Drummen NE, Smit E, Vermeer C and Theuwissen E: Three-year low-dose menaquinone-7 supplementation helps decrease bone loss in healthy postmenopausal women. Osteoporos Int 24: 2499-2507, 2013.

2. Koshihara Y, Hoshi K, Ishibashi H and Shiraki M: Vitamin K2 promotes 1alpha, $25(\mathrm{OH}) 2$ vitamin $\mathrm{D} 3$-induced mineralization in human periosteal osteoblasts. Calcif Tissue Int 59: 466-473, 1996.

3. Iwamoto J, Matsumoto H and Takeda T: Efficacy of menatetrenone (vitamin K2) against non-vertebral and hip fractures in patients with neurological diseases: Meta-analysis of three randomized, controlled trials. Clin Drug Investig 29: 471-479, 2009.

4. Cockayne S, Adamson J, Lanham-New S, Shearer MJ, Gilbody S and Torgerson DJ: Vitamin K and the prevention of fractures: Systematic review and meta-analysis of randomized controlled trials. Arch Intern Med 166: 1256-1261, 2006.

5. Akiyama Y, Hara K, Kobayashi M, Tomiuga T and Nakamura T: Inhibitory effect of vitamin $\mathrm{K} 2$ (menatetrenone) on bone resorption in ovariectomized rats: A histomorphometric and dual energy X-ray absorptiometric study. Jpn J Pharmacol 80: 67-74, 1999.

6. Azuma K, Casey SC, Ito M, Urano T, Horie K, Ouchi Y, Kirchner S, Blumberg B and Inoue S: Pregnane X receptor knockout mice display osteopenia with reduced bone formation and enhanced bone resorption. J Endocrinol 207: 257-263, 2010. 
7. Fu X, Moreines J and Booth SL: Vitamin K supplementation does not prevent bone loss in ovariectomized Norway rats. Nutr Metab (Lond) 9: 12, 2012.

8. Landeira-Fernandez J: Analysis of the cold-water restraint procedure in gastric ulceration and body temperature. Physiol Behav 82: 827-833, 2004

9. Huang P, Zhou ZR, Zheng MQ and Shi FX: Effect of the IGF-1/PTEN/Akt/FoxO signaling pathway in the duodenal mucosa of rats subjected to water immersion and restraint stress. Genet Mol Res 11 (AOP): 4775-4788, 2012.

10. Szlachcic A, Sliwowski Z, Krzysiek-Maczka G, Majka J, Surmiak M, Pajdo R, Drozdowicz D, Konturek SJ and Brzozowski T: New satiety hormone nesfatin-1 protects gastric mucosa against stress-induced injury: Mechanistic roles of prostaglandins, nitric oxide, sensory nerves and vanilloid receptors. Peptides 49: 9-20, 2013.

11. Nur Azlina MF, Kamisah Y, Chua KH and Qodriyah HM: Tocotrienol attenuates stress-induced gastric lesions via activation of prostaglandin and upregulation of COX-1 mRNA. Evid Based Complement Alternat Med 2013: 804796, 2013.

12. Magierowski M, Jasnos K, Pawlik M, Krzysiek-Maczka G Ptak-Belowska A, Olszanecki R, Kwiecien S, Korbut R and Brzozowski T: Role of angiotensin-(1-7) in gastroprotection against stress-induced ulcerogenesis. The involvement of mas receptor, nitric oxide, prostaglandins and sensory neuropeptides. J Pharmacol Exp Ther 347: 717-726, 2013.

13. Ohta Y, Yashiro K, Ohashi K and Imai Y: Disruption of non-enzymatic antioxidant defense systems in the brain of rats with water-immersion restraint stress. J Clin Biochem Nutr 51: 136-142, 2012

14. Takeda T, Hosokawa M, Takeshita S, Irino M, Higuchi K Matsushita T, Tomita Y, Yasuhira K, Hamamoto H and Shimizu K: A new murine model of accelerated senescence. Mech Ageing Dev 17: 183-194, 1981.

15. Matsushita M, Tsuboyama T, Kasai R, Okumura H, Yamamuro T, Higuchi K, Higuchi K, Kohno A, Yonezu T, Utani A, et al: Age-related changes in bone mass in the senescence-accelerated mouse (SAM). SAM-R/3 and SAM-P/6 as new murine models for senile osteoporosis. Am J Pathol 125: 276-283, 1986.

16. Ohnishi K, Tomimoto H, Akiguchi I, Seriu N, Kawamata T, Nakamura S, Kimura J, Nishio T, Higuchi K and Hosokawa M: Age-related decrease of nerve growth factor-like immunoreactivity in the basal forebrain of senescence-accelerated mice. Acta Neuropathol 90: 11-16, 1995.

17. Takeda T, Hosokawa M and Higuchi K: Senescence-accelerated mouse (SAM): A novel murine model of senescence. Exp Gerontol 32: 105-109, 1997.

18. Washimi Y, Chen H, Ito A, Takao R, Uzawa T, Yamamoto Y, Yamada $\mathrm{H}$ and Shoumura S: Effect of intermittent treatment with human parathyroid hormone 1-34 in SAMP6 senescence-accelerated mice. J Encocrinol Invest 33: 395-400, 2010.

19. Takagi $\mathrm{K}$ and Okabe S: The effects of drugs on the production and recovery processes of the stress ulcer. Jpn J Pharmacol 18 : 9-18, 1968

20. Parfitt AM: The cellular basis of bone remodeling: The quantum concept reexamined in light of recent advances in the cell biology of bone. Calcif Tissue Int 36 (Suppl 1): 37-45, 1984.

21. Kawamori Y, Katayama Y, Asada N, Minagawa K, Sato M, Okamura A, Shimoyama M, Nakagawa K, Okano T, Tanimoto M, et al: Role for vitamin D receptor in the neuronal control of the hematopoietic stem cell niche. Blood 116 : $5528-5535,2010$
22. Dempster DW, Compston JE, Drezner MK, Glorieux FH, Kanis JA, Malluche H, Meunier PJ, Ott SM, Recker RR and Parfitt AM: Standardized nomenclature, symbols and units for bone histomorphometry: A 2012 update of the report of the ASBMR Histomorphometry Nomenclature Committee. J Bone Miner Res 28: 2-17, 2013.

23. Duque G, Macoritto M, Dion N, Ste-Marie LG and Kremer R: $1,25(\mathrm{OH}) 2 \mathrm{D} 3$ acts as a bone-forming agent in the hormone-independent senescence-accelerated mouse (SAM-P/6). Am J Physiol Endocrinol Metab 288: E723-E730, 2005.

24. Tomita M, Katsuyama H, Okuyama T, Watanabe Y, Hidaka K, Otsuki T and Nata M: The effect of CAG repeat polymorphism in the glucocorticoid receptor on stress responses of mice exposed to water-immersion restraint stress. Int J Mol Med 25: 415-420, 2010.

25. Sasaki H, Miyakoshi N, Kasukawa Y, Maekawa S, Noguchi H, Kamo K and Shimada Y: Effects of combination treatment with alendronate and vitamin $\mathrm{K} 2$ on bone mineral density and strength in ovariectomized mice. J Bone Miner Metab 28 403-409, 2010

26. Tomimori Y, Mori K, Koide M, Nakamichi Y, Ninomiya T, Udagawa $\mathrm{N}$ and Yasuda $\mathrm{H}$ : Evaluation of pharmaceuticals with a novel 50-hour animal model of bone loss. J Bone Miner Res 24: 1194-1205, 2009.

27. Sakata T, Sakai A, Tsurukami H, Okimoto N, Okazaki Y, Ikeda S, Norimura T and Nakamura T: Trabecular bone turnover and bone marrow cell development in tail-suspended mice. J Bone Miner Res 14: 1596-1604, 1999.

28. Ohsaki Y, Shirakawa H, Miura A, Giriwono PE, Sato S, Ohashi A, Iribe M, Goto T and Komai M: Vitamin K suppresses the lipopolysaccharide-induced expression of inflammatory cytokines in cultured macrophage-like cells via the inhibition of the activation of nuclear factor $\kappa \mathrm{B}$ through the repression of IKK $\alpha / \beta$ phosphorylation. J Nutr Biochem 21: 1120-1126, 2010.

29. Ichikawa T, Horie-Inoue $K$, Ikeda $K$, Blumberg $B$ and Inoue $S$ : Vitamin K2 induces phosphorylation of protein kinase A and expression of novel target genes in osteoblastic cells. J Mol Endocrinol 39: 239-247, 2007.

30. Iwamoto J, Matsumoto H, Tadeda T, Sato Y and Yeh JK: Comparison of the effect of vitamin $\mathrm{K}(2)$ and residronate on trabecular bone in glucocorticoid-treated rats: A bone histomorphometry study. Yonsei Med J 50: 189-194, 2009.

31. Katsuyama H, Saijoh K, Otsuki T, Tomita M, Fukunaga M and Sunami S: Menaquinone-7 regulates gene expression in osteoblastic MC3T3E1 cells. Int J Mol Med 19: 279-284, 2007.

32. Atkins GJ, Welldon KJ, Wijenayaka AR, Bonewald LF and Findlay DM: Vitamin K promotes mineralization, osteoblast-to-osteocyte transition and an anticatabolic phenotype by \{gamma\}-carboxylation-dependent and -independent mechanisms. Am J Physiol Cell Physiol 297: C1358-C1367, 2009.

33. Koshihara Y, Hoshi K, Okawara R, Ishibashi H and Yamamoto S: Vitamin $\mathrm{K}$ stimulates osteoblastogenesis and inhibits osteoclastogenesis in human bone marrow cell culture. J Endocrinol 176: $339-348,2003$ 